\title{
Maximum Traffic Scheduling and Capacity Analysis for IEEE 802.15.3 High Data Rate MAC Protocol
}

\author{
Yi-Hsien Tseng*, Eric Hsiao-kuang $\mathrm{Wu}^{\dagger}$ and Gen-Huey Chen* \\ *Department of Computer Science and Information Engineering, National Taiwan University, Taipei, Taiwan. \\ Email: yhtseng@inrg.csie.ntu.edu.tw, ghchen@csie.ntu.edu.tw \\ ${ }^{\dagger}$ Department of Computer Science and Information Engineering, National Central University, Taipei, Taiwan. \\ Email: hsiao@csie.ncu.edu.tw
}

\begin{abstract}
IEEE 802.15.3 is designed to provide low complexity, low cost, low power consumption and high data rate wireless connectivity among devices within wireless personal area networks (WPAN). A piconet is a basic topology of WPAN and is defined as a collection of one or more associated devices that share a single piconet coordinator (PNC). One functionality of a PNC is to schedule traffic of a piconet. But the scheduling method of a PNC is out of the scope of IEEE 802.15.3 draft standard. This paper proposes a scheduling method which is called Maximum Traffic (MT) scheduling for IEEE 802.15.3. MT scheduling guarantees that total transmission data is maximum at any point of time and the needed time slots for transmission is minimum. MT scheduling is designed and analyzed by the properties of a graph coloring problem. Furthermore, the reasonable number of devices for a piconet and the durations of Contention Access Period (CAP) and Contention Free Period (CFP) are also investigated by this paper.
\end{abstract}

\section{INTRODUCTION}

IEEE 802.15.3 [1] draft standard defines the PHY and MAC specifications for high data rate wireless networks. The MAC protocol of IEEE 802.15.3 supports both isochronous and asynchronous data types and is designed to support additional physical layers such as UWB [4][8]. The 802.15.3 MAC mainly works within a piconet. A piconet is defined as a wireless ad hoc data communications system which allows a number of independent data devices (DEVs) to communicate with each other. The DEV is the basic component of a piconet. One DEV is required to be a piconet coordinator (PNC) of a piconet. The PNC provides the basic timing and additional information for a piconet via the beacon which is broadcasted at the beginning of a superframe period.

The PNC broadcasts the information of all of the DEVs periodically and updates information for the new DEV in the beacon when a new DEV joins a piconet. This allows other DEVs in the piconet to be aware of the new DEV and introduces the new DEV about the members of the piconet as well. The 802.15.3 MAC provides a hand over function for the transition of the old PNC to the new PNC. When a DEV associates with an existing piconet, the PNC checks the capabilities of the new DEV. If the new DEV is more capable, then the PNC has the option of handing over control of the piconet to the DEV that has just joined.

Timing for data transmission in the piconet is based on the superframe. A superframe consists of three parts:
1) The beacon: Set the timing allocations and communicate management information for the piconet.

2) The contention access period (CAP): Communicate commands and/or asynchronous data if it is present in the superframe. The CSMA/CA protocol is applied in this window.

3) The contention free period (CFP): Communicate commands, isochronous streams and asynchronous data. This window is TDMA based.

The length of CAP is dynamic and determined by the PNC. All data in the piconet is exchanged in a peer-to-peer manner. The DEVs communicate data either in CAP or CFP. If a DEV needs channel time from $\mathrm{CFP}$, it makes a request from the PNC for channel time (i.e. CFP time slot). Then the PNC allocates time in a guaranteed time slot (GTS) for the DEV as the resources are available. In other words, all communicating data in CFP must be allocated by the PNC. Furthermore, the 802.15.3 MAC provides a functionality of controlling transmit power. The PNC is allowed to determine a maximum transmit power for CAP and beacon; meanwhile, all DEVs are allowed to calculate individual minimum transmit powers to reach the target DEVs.

Since IEEE 802.15.3 is designed to support new generation physical layers such as UWB which offers the precise position location, this paper assumes that a PNC can calculate the relative positions [3][5] of all DEVs of a piconet. By this assumption, we propose a MT scheduling scheme which guarantees maximum traffic and minimum needed time slots. In addition, this paper gives the analysis for the duration of CAP and GTS (Guaranteed Time Slot). The main contributions are including: (1) the determination of the upper bound of required time slots for a traffic-saturated network and (2) the quantitative analysis which is adopted to determine the lengths of CAP.

\section{Motivations}

Different scheduling methods carry distinct criterions. This paper proposes the Maximal Traffic (MT) scheduling scheme which is based on two criterions. First, the total transmission data is maximal at any point of time. The other one is that the number of time slots of a superframe is the least.

The main idea of the MT scheduling is illustrated in Figure 1. Suppose that there are four links to be scheduled, and three 


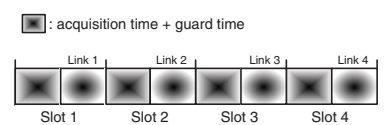

(A)

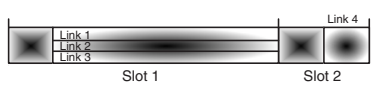

(B)
Fig. 1. Benefits of MT scheduling: (A) general scheduling vs. (B) MT scheduling.

of them are independent transmit-receive pairs (could transmit simultaneously without causing interference). Figure 1.(A) is a general scheduling, and we can see that the required number of time slots for transmission is four. Furthermore, the time for channel acquisition and TDMA guard time is 4 time units. The MT scheduling puts the independent transmit-receive pairs into the same time slot and could extend the transmission length according to the number of independent pairs which are in the same time slot (Figure 1.(B)). The time for channel acquisition and TDMA guard time will be reduced to two time units and the time slot length for three independent pairs is increased to three times of the original one.

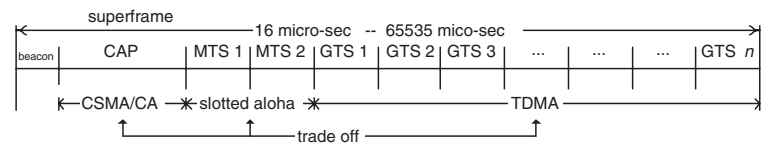

Fig. 2. The superframe of 802.15.3 MAC.

The superframe of IEEE 802.15.3 is illustrated as Figure 2. MTSs are used for control information exchanges between DEVs and the PNC. GTSs are utilized for traffic communications between the DEVs. The access protocol for MTS is slotted aloha that provides more quick access than CSMA/CA. The more length of CAP or MTS is, the more DEVs can submit requirements for slots. However, it causes less time slots for transmitting. Therefor, analyzing the optimal lengths of CAP, MTS and GTS is critifcal for IEEE 802.15.3. The main purpose of MTS is designed to support quick registration and is out of the scope of this paper. Therefor we focus on giving probability models to analyze the length of CAP during which only channel request packets are allowed to be exchanged.

\section{RELATED WORK AND ANALYSis MODEL}

This section describes and derives a probability model for CAP of IEEE 802.15.3. First, a previous work [2] for CSMA/CA of IEEE 802.11 is introduced. And we extend the results of the previous work for the framework of our model. Second, the backoff procedure of CAP is described. This backoff procedure of CAP is similar to CSMA/CA of IEEE 802.11. Furthermore, the more detailed parameters of CAP are shown as well. And we derive the probability model of CAP finally. Although the mechanism of CAP is the same as CSMA/CA of IEEE 802.11. But the duration of CAP is limited and is different from CSMA/CA of IEEE 802.11.

\section{A. IEEE 802.11 Capacity Analysis}

The distributed coordination function (DCF) which is a CSMA/CA MAC protocol is a basic access method of IEEE 802.11. The paper [2] proposes a conscientious and careful probability model for maximizing the capacity of DCF. This subsection introduces some basic models [2] in order to derive our model.

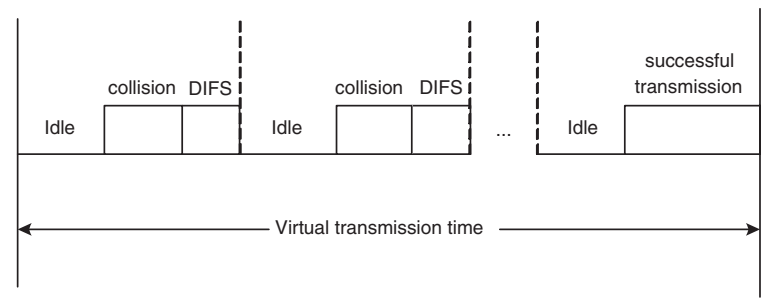

Fig. 3. IEEE 802.11 channel view of a station transmit a packet successfully.

Figure 3 shows a channel view of the required time (which is called virtual transmission time in [2]) that a station can transmit a packet successfully. And the average required time is denoted as the following:

$t_{v}=E\left[\sum_{i=1}^{N_{c}}\left(I_{d l e_{-}} p_{i}+\operatorname{Coll}_{i}+\tau+D I F S\right)\right]+E\left[I d l_{-} p_{N_{c}+1}\right]+E[S]$

And can be rewritten as

$t_{v}=E\left[N_{c}\right]\{E[C o l l]+\tau+D I F S\}+E\left[I_{d l e_{p}}\right] \cdot\left(E\left[N_{c}\right]+1\right)+E[S]$

where

$N_{c}$ : the number of collisions in a virtual time.

Idle_ $p_{i}$ : the length of the $i$ th idle period.

$\mathrm{Coll}_{i}$ : the length of the $i$ th collision time.

$\tau$ : the maximum propagation time.

$S$ is the time required to complete a successful transmission without any collision and backoff time. It follows that

$$
S \leq m+2 \tau+S I F S+A C K+D I F S
$$

Furthermore, for calculating $t_{v}$, the distribution functions of $N_{c}, I d l e_{-} p_{i}$, and $\operatorname{Coll}_{i}$ must be known. Since we allow that the channel request packet can be transmitted in CAP, the length of collision (i.e. Coll $_{i}$ ) of our model is constant. Therefor, only distribution functions of $N_{c}$ and $I d l e_{-} p_{i}$ are listed as the following:

$M$ : the number of stations.

$p$ : the probability of a station can transmit in a slot.

- $N_{c}$ :

$$
P\left\{N_{c}=i\right\}=P_{\text {Coliision }}^{i} \cdot P_{\text {Success }}
$$

where

$P_{\text {Collision }}=P\{$ transmitting stations $\geq 2 \mid$

transmitting stations $\geq 1\}$

$$
=\frac{1-(1-p)^{M}-M p(1-p)^{M-1}}{1-(1-p)^{M-1}}
$$


$P_{\text {Success }}=P\{$ transmitting stations $=1 \mid$

transmitting stations $\geq 1\}$

$$
=\frac{M p \cdot(1-p)^{M-1}}{1-(1-p)^{M-1}}
$$

Thus,

$$
E\left[N_{c}\right]=\frac{1-(1-p)^{M}}{M p(1-p)^{M-1}}-1
$$

-Idle_p $p_{i}$ :

$\mathrm{P}\{0$ transmitting station in a slot $\}$

$$
=(1-p)^{M}
$$

$\mathrm{P}\{$ at least 1 transmitting stations in a slot\}

$$
=1-(1-p)^{M}
$$

Thus,

$$
E\left[\text { Idle }_{p}\right]=t_{\text {slot }} \cdot\left[1-(1-p)^{M}\right] \cdot \sum_{i=1}^{\infty} i\left[(1-p)^{M}\right]^{i}
$$

\section{B. Backoff Procedure of CAP}

The carrier sense multiple access with collision avoidance (CSMA/CA) is the basic medium access mechanism during CAP. The types of packets which are allowed to transmit are determined by the PNC. And a DEV is allowed to transmit one frame at a time with a backoff applied. The DEV shall abort the transmission if there is not enough room for this entire frame exchange sequence.

The backoff algorithm of CAP is the same as basic CSMA/CA (i.e. without RTS/CTS) of IEEE 802.11. But there are several different parameter setups between them. The main information which is used by the backoff algorithm of CAP is shown as follows,

- retry_count: A retry count that takes on values in the range $[0,3]$.

- backoff_window(retry_count): A table which has values $[7,15,31,63]$.

- bw_random(retry_count): A random integer which is selected from a uniform distribution over the interval [0,backoff_window(retry_count)].

Before initiating the backoff algorithm, the DEV first waits a RIFS duration. When the medium is determined to be idle during a RIFS duration, the DEV shall choose backoff_count = bw_random(retry_count) to start backoff. The backoff counter shall be suspended whenever the channel is busy. A frame can be transmitted until the backoff counter is set to 0 . The retry_count shall be incremented but shall not be set to more than 3 when a directed frame is transmitted and the expected $\mathrm{ACK}$ is not correctly received by the DEV.

\section{Probability Model of CAP}

The capacity analysis of this paper focus on the relationship between the duration of CAP and the number of stations which are ready to transmit a channel request packet. This restriction is reasonable because PNC can determine it.
First, we denote a random variable $t_{s}$ to represent the time of a station transmitting a packet successfully. Immediately, the equation (1) is the mean of the $t_{s}$ while the RIFS is applied instead of DIFS. Since only channel request packets are allowed to transmit, the $\mathrm{Coll}_{i}$ and the $S$ are both constant. Now, we want to derive the probability (denoted by $P_{M}\left\{t_{s}=\right.$ $j$ ) ) of a station of $M$ stations to transmit a packet successfully when the duration of CAP is $j$. Because the $\mathrm{Coll}_{i}$ and the $S$ are both constant, the duration of CAP $j$ minus $E\left[N_{c}\right]$ times the length of $\operatorname{Coll}_{i}$ and a $S$ is the time of $E\left[N_{c}\right]+1$ times the length of $E\left[\operatorname{Idel}_{p}\right]$. From (8) and (9), the distribution function of $I_{d l e}$ follows that

$$
P\left\{\text { Idle }_{p}=k \text { slots }\right\}=\left((1-p)^{M}\right)^{k} \cdot\left(1-(1-p)^{M}\right)
$$

And from (5) and (6), the average number of collisions when the duration of CAP is $j$ follows that

$$
\begin{aligned}
& E\left[N_{c_{-} j}\right]= \\
& \sum_{i=0}^{j}\left(\frac{1-(1-p)^{M}-M p(1-p)^{M-1}}{1-(1-p)^{M-1}}\right)^{i} \cdot\left(\frac{M p \cdot(1-p)^{M-1}}{1-(1-p)^{M-1}}\right)
\end{aligned}
$$

From the channel view of a virtual transmission time, we can assume that each idle time is uniform distribution over the sum of all idle times. It thus follows that

Idel_Time $=\frac{j-\left(L_{\text {collision }}+\tau+R I F S\right) \cdot E\left[N_{c_{-} j}\right]-L_{S}}{E\left[N_{c_{-} j}\right]+1}$

where

$L_{\text {collision }}$ : the length of collision is a constant.

$L_{S}$ : the length of transmitting a packet successfully without any collision and idle is also a constant.

A DEV of $M$ DEVs can complete a transmission successfully if and only if the idle time of each $i d l e_{p}$ no more than Idel_Time. Therefore, the probability of $P_{M}\left\{t_{s}=j\right\}$ can be derived by the product of the probability of each Idle $_{p} \leq$ Idel_Time and follows that

$$
\left.P_{M}\left\{t_{s}=j\right\}=\prod_{i=1}^{\left\lceil E\left[N_{c_{-} j}\right]\right\rceil+1\left\lceil\frac{\text { Idle_Time }}{\text { aslot } T \text { ime }}\right\rceil} P\left\{\sum_{k=0}^{\text {Idle }} e_{p}=k \text { slots }\right\}\right)
$$

With (14) we are now able to determine the duration of CAP. The $M$ and $p$ are two parameters which are used to compute $t_{s}$, and $p$ depends on $M$. Thus, a PNC can setup the length of CAP based on the number of DEVs $(M)$.

\section{Maximum Traffic (MT) Scheduling}

All traffics that occur in a CFP window must be controlled by the PNC. Different scheduling methods carry the different criterions. This paper proposes the Maximal Traffic (MT) scheduling which is based on two criterions. First, the total transmission data is maximal at any point of time. And another one is that the number of time slots of a superframe is the least.

As a DEV requests channel time to transmit, the DEV submits a request to the PNC. The request includes ids (identifications) of the sender and the receiver, required information defined in IEEE 802.15.3 draft standard and the 


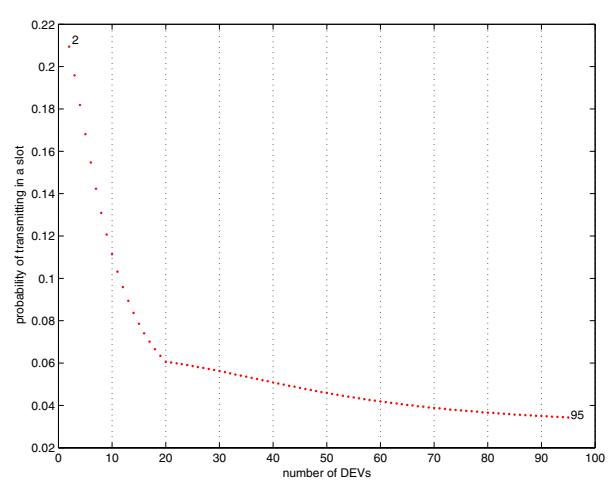

(a)

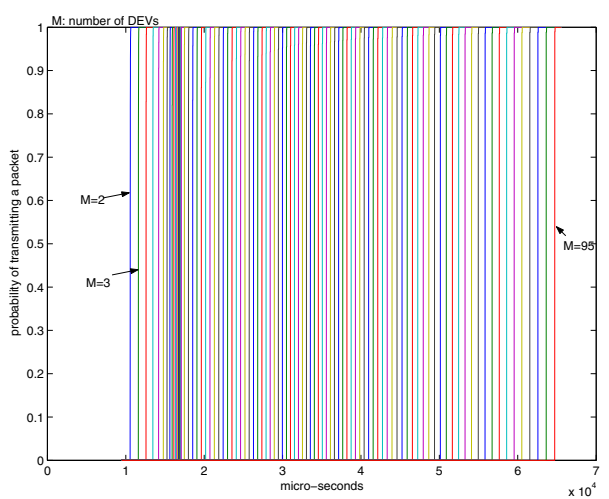

(b)

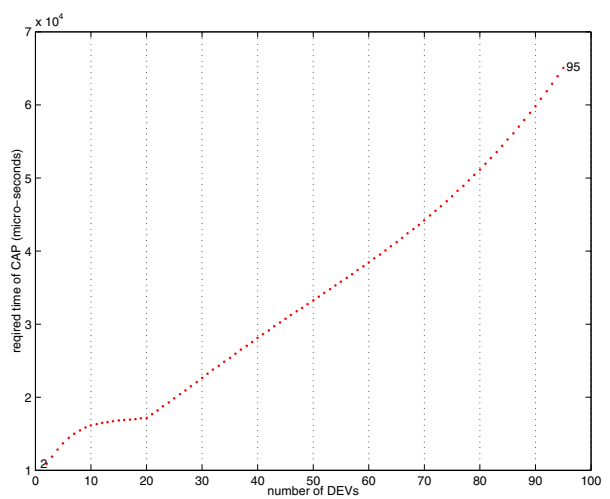

(c)

Fig. 4. Numerical results of capacity analysis for CAP. (a) Probability of a DEV which can start to transmit successfully in a slot. (b) Transmission probability of a DEV when there are M DEVs in a piconet. (c) Required time of a DEV which can transmit a packet with probability near 1.

ids of all DEVs which are interfered by this transmit-receive pair especially. The information about interfered DEVs is calculated by the sender via the relative position information. The scheduling problem for the PNC is described formally in Problem 1.

Problem 1: Given some transmitting pairs, group these pairs such that the pairs in the same transmitting group can transmit in the same time slot simultaneously and the number of groups is minimal.

For solving this problem, we model and reduce Problem 1 to the graph coloring problem. A $k$-coloring of a graph is defined as an assignment of one of $k$ distinct colors to each vertex so that no two adjacent vertices are given the same color. And the chromatic number is the smallest $k$ such that the graph can be $k$-colored. First, we give the definition of our graph.

Definition 1: Consider a graph $G=(V, E)$

1) Re-denote by $V$ the set of transmit-receive pairs.

2) Denote that there is an edge $e \in E$ between $v_{i}$ and $v_{j}$ $\left(v_{i}, v_{j} \in V\right)$ iff either $v_{i}$ 's receiver is within $v_{j}$ 's transmitting range or $v_{j}$ 's receiver is within $v_{i}$ 's transmitting range.

The Problem 1 can be solved through finding chromatic number from the graph of Definition 1. There are some algorithms for finding chromatic number in [6]. The properties of chromatic number guarantee that the number of needed time slots is minimum. The vertices (transmit-receive pairs) with same color can be put into the same group (time slot) and can be allowed to transmit simultaneously.

\section{Simulation and Numerical Results}

As mentioned in section III, the probability model (14) can be adopted to determine the length of CAP according to the number of DEVs in a piconet. The numerical relationship between the length of CAP and the number of DEVs is given in subsection A. As well as MT scheduling is analyzed in subsection B by combination of simulation and mathematical analysis.

\section{A. Capacity of CAP}

Before being able to calculate the probability (14), the value $p$ must be known. The iterative algorithm which is proposed in [2] can be used to compute $p$. The main factor of computing $p$ is contention window (CW) size. The CW size of IEEE 802.15.3 has been described in the previous section and be taken into the iterative algorithm. The values $p$ of variance number of DEVs are shown as Fig. 4(a). The maximal number of DEVs in Fig. 4(a) is 95 since the maximal capacity of CAP is 95 (DEVs) and will be illustrated later.

TABLE I

IEEE 802.15.3 PARAMETERS

\begin{tabular}{|c|c|}
\hline a backoff slot time & $5.8 \mu \mathrm{sec}$ \\
\hline$\tau$ & $1 \mu \mathrm{sec}$ \\
\hline RIFS & $15.8 \mu \mathrm{sec}$ \\
\hline ACK & $532.7 \mu \mathrm{sec}$ \\
\hline channel time request packet (CTR) & $1420.5-7013.5 \mu \mathrm{sec}$ \\
\hline PHY preamble & $887.8 \mu \mathrm{sec}$ \\
\hline PHY header & $88.8 \mu \mathrm{sec}$ \\
\hline
\end{tabular}

Now, it is ready to compute the probability of a DEV which can transmit a packet successfully when there are $M$ DEVs in a piconet. Table I are the required parameters of IEEE 802.15.3 that are needed for computing (14). The results are shown in Fig. 4(b). From these results we can see that the maximal number of DEVs is 95 because the maximal duration of superframe is $65535 \mu \mathrm{sec}$. It means that DEVs have very low probability (near 0) that anyone of these DEVs can transmit a packet when the number of DEVs is over 95.

Furthermore, we want to get the required time that a DEVs can transmit a channel request packet successfully when the number of DEVs in a piconet is $M$. The required time is derived by taking the time $t$ satisfies that it is the first $t$ such that $\left[1-P_{M}\left(t_{s}=t\right)\right]<\epsilon$. The $\epsilon$ is defined as a very small 
value and is $2.2204 e-016$. According to the above method, the required time can be computed and be drawn in Fig. 4(c). The results in Fig. 4(c) can help to determine the length of CAP of superframe by calculating the sum of required time based on number of DEVs.

\section{B. Analysis of MT scheduling for CFP}

Via reviewing the Definition 1, it is clear that the less edges are in the graph, the less chromatic number is needed. First, we analyze the worst case of interference by simulation. There are two types of acknowledgement types in the simulation; acknowledgement (ACK) and no acknowledgement (no-ACK). The ACK type causes more interference than the no-ACK type. All DEVs are generated randomly on a $10 \mathrm{~m} \times 10 \mathrm{~m}$ area and the position of the PNC is also set randomly. For transmission, Each DEV randomly chooses one of any other DEVs to communicate. Thus, this is the most congested scenario for a piconet. Then we model these topologies to the corresponding graph by Definition (1) and calculate the ratio of exact interference (edges) caused by exact links to the edges of complete graph. The results are showed in Figure 5.

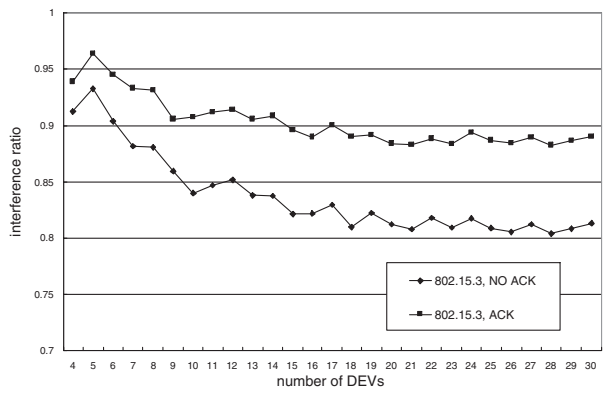

Fig. 5. Interference ratio

Furthermore, we analyze the least time slots that can be decreased according to the results of the interference ratio. If the minimum chromatic number of an $n$-vertices graph is $m$ and $m<=n$, then the edges of the graph are $C_{2}^{m}$ at least[7]. Therefore, by this concept, obtaining the most chromatic number (the most number of time slots) from the results of interference ratio is easy. For example, the chromatic number for a graph with 9 edges is at most 4 because $C_{2}^{4}=$ $6<9<10=C_{2}^{5}$. The results are shown in Figure 6 .

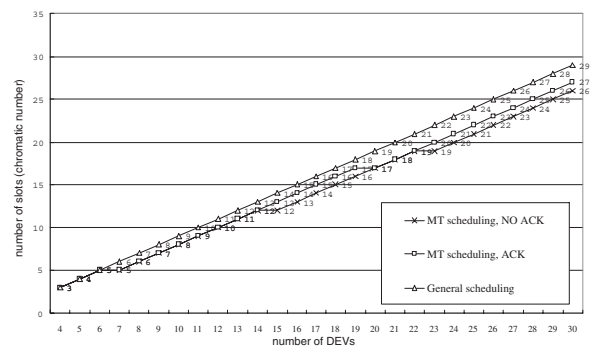

Fig. 6. Upper bound of required time slots

\section{CONCLUSION}

IEEE 802.15.3 is a new standard that specifies high-speed, short range and low energy consumption wireless communication technology. IEEE 802.15.3 is a central architecture and needs a PNC to manage the traffic of a piconet. In this paper, we propose a schedule method and make a capacity analysis of CAP. Both of them are useful for practical implementation.

First, a probability model of CAP is derived according to previous work [2]. This model is applied to decide the length of CAP based on the number of DEVs. From the results of capacity analysis for CAP, we can see that a piconet can allow at most $95 \mathrm{DEV}$ s to join when the duration of CAP is maximal (near $65535 \mu \mathrm{sec}$ which depends on the length of beacon packet). Also, adopting the results contributes to determining the duration of CAP based on existing traffic requirements and the number of DEVs. Second, we model and reduce the scheduling problem to a graph problem (graph coloring problem). We design a MT scheduling method and analyze the performance of MT scheduling via applying the concept of the graph coloring. The analysis of MT scheduling shows that the required time slots of CFP can be reduced one or two slots at least when the traffic of piconet is most saturated. Furthermore, MT scheduling can be cooperated with any other scheduling methods in order to support QoS requirements.

The current analysis of this paper focuses on one piconet. Further research could be extended to more than one piconet. In addition, finding an optimal number of DEVs in a piconet and transmitting data over multi-piconets by adopting relative position information are also key issues for forming a large scale UWB ad hoc networks.

\section{ACKNOWLEDGMENT}

This work was supported in part by the MediaTek Inc. This work was also supported by the Communications Software Technology project of Institute for Information Industry and sponsored by MOEA, R.O.C.

\section{REFERENCES}

[1] IEEE Draft Std 802.15.3: Wireless Medium Access Control (MAC) and Physical Layer (PHY) Specifications for High Rate Wireless Personal Area Networks, Inst. Elec. Electron. Eng., New York, USA, June, 2002.

[2] F. Cali, M. Conti and E. Gregori, "Dynamic tuning of the IEEE 802.11 protocol to achieve a theoretical throughput limit," in IEEE/ACM transactions on networking, 2000, Vol. 8, pp. 785-799.

[3] S. Capkun, M. Hamdi and J. P. Hubaux, "GPS-free positioning in mobile ad-hoc networks," in proceeding of the 34th Annual Hawaii International Conference, 2001, pp. 3481-3490.

[4] J. Foerster, E. Green, S. Somayazulu and D. Leeper, "Ultra-wideband technology for short- or medium-range wireless communications," in Intel technology journal, 2nd quarter, 2001.

[5] R. J. Fontana and S. J. Gunderson, "Ultra-wideband precision asset location system," on Conf. Ultra Wideband Systems and Technologies, 2002, pp. 147-150.

[6] X. Y. Li and Y. Wang, "Simple heuristics and PTASs for intersection graphs in wireless ad hoc networks," in Proc. The 6th international workshop on Discrete algorithms and methods for mobile computing and communications, September, 2002, pp. 62-71.

[7] D. B. West, Introduction to Graph Theory, Pretice Hall Inc., Upper Saddle River, NJ, 2001.

[8] M. Z. Win and R. A. Scholtz, "Impulse radio: How it works," IEEE Commun. Lett., February, 1998, vol. 2, pp. 36-38. 